\title{
Experimental tests in human-robot collision evaluation and characterization of a new safety index for robot operation
}

\author{
Cristina Alén Cordero Giuseppe Carbone Marco Ceccarelli \\ Javier Echávarri José Luis Muñoz
}

\begin{abstract}
A B S T R A C T
This paper describes an experimental procedure consisting of impact tests that simulate a collision of a human head with an industrial robot with the aim to validate a safety index named as New Index for Robots (NIR) and its outputs. The experiments in this paper are based on lab tests. It is an attempt to characterize the NIR index underlying the main parameters that are involved in crash interaction and to highlight limitations and weakness of suggested impact tests.
\end{abstract}

\section{Introduction}

The term "service robot" that was tentatively defined by the International Federation of Robotics (IFR) as "a robot which operates semi or fully autonomously to perform services useful to the well being of humans and equipment, excluding manufacturing operations" has now a more adjusted definition included in recently approved ISO 8373: 2012 Robots and robotic devices: vocabulary. According to this new vocabulary, a "service robot" is a robot that performs useful tasks for humans or equipment excluding industrial automation applications and it can be distinguished between service robot for personal use (used for a non-commercial task, by a lay person) and service robot for professional use (used for a commercial task, by properly trained operator) [1].

The close interaction among service robots and humans makes safety constraints one of the most significant aspects of robot design and operation, including not only the aim of avoiding collision, but also of investigating and minimizing consequences of collisions, that are caused by fast or unforeseen movements of robots. 
Although reduction of collision consequences has been widely explored in the field of safety in automotive (performing crash tests by using different models to simulate human body), the results of car industry cannot be directly extrapolated to impact studies among robots and humans. Nevertheless it can be used as a good base to compare with.

Intrinsic safety design in robots for industrial use is still a matter of discussion in industrial manufacturing fields, where mechanical risks have been traditionally avoided by using adequate protections that are linked either to robot design or indirectly to the robot operational process, like for example by putting physical barriers between robot and operators.

However, this situation changes as long as there is closer interaction between human and robots. Even if some standards $[2,3]$ define new collaborative operation requirements for industrial robots (minimal speed, maximum dynamic power or maximum static force), the lack of specific standards for service robots needs to be covered by new viewpoints [4]. In general a service robot is understood as a robotic system with a certain level of autonomy in performing service operations for given tasks within a specified environment and interaction with human users, while industrial robots are devoted to predefined operations in industrial tasks that are very often well-structured environments [2]. Peculiarities for design and operation of service robots are outlined in [5] with a special attention also to safety issues much more than in industrial robots.

Safety is then an issue of increasing importance mainly when robots interact or collaborate with human users. Basic rules for industrial robots, as in [2] needs to be enlarged when humans may have risk in interaction with robots. Main factors for safety issues are related with risks of impact of robot part with human body or part of it, not only in malfunctioning of the systems but more and more in the operations with strict interaction between robot and human operators, as reported in [4].

Several strategies have been developed to study biomechanics in the brain injury [6], through experimental, mathematical or observational approaches, and to link it to appropriate safety criteria [6-9]. According to those theories, which were based on the experience of crash tests in automotive industry, several indexes have been empirically formulated to link quantitative injuries with safety levels of robots. Examples of these indexes in Robotics are described in [10-12].

But the main limit of these extrapolated indexes can be considered that most of them have been mainly evaluated by means of mathematics or simulation programs (LS-DYNA) [13,14] whereas only a few have been assessed by using test devices [15] (first experimental evaluation of HIC, at an automobile certified crash-test facility using DLR lightweight robot III and a Hybrid III dummy). The need of using experimental measure in safety evaluation of robot operation has been also stressed in [16] where impact tests with a seven-degree of freedom flexible joint robot (Lightweight Robot III) of $14 \mathrm{~kg}$ against a Hybrid III crash test Dummy were performed.

The aim of the experiment reported in this paper is to setup an appropriate scenario to reproduce a basic collision between industrial robot and human in a proper way so that the outcomes can be used to check the implementation of the New Index for Robots [17], which is one of aforementioned safety indexes in robotics field, with a comparison with one of the most globally recognized index in [18]. Moreover, one of the contributions of the reported experiments is an experimental evaluation of the proposed new index for robots in a proper testing frame with suitable setup and sensors.

The appropriate scenario has been conceived by looking at the main aspects of impact and robot actions together with possibility of a fairly simple laboratory set-up for tests that have been performed also for a validation of the proposed new safety index. This fairly simple laboratory set-up has been arranged with details that are reported in [15]. The characteristics of the laboratory set-up can be summarized in using an industrial robot as the available ADEPT SCARA robot with fairly simple models of human body parts with sensors that are installed both on the robot arm and model surfaces.

The rest of the article is structured in the following way. Section 2 introduces different approaches that were developed from earlier 1960s of safety indexes. It also introduces the first indexes proposed in robotic fields. An experiment lay out that was setup to perform the tests is described in Section 3, where software \& hardware and experiment restrictions, such as speed conditions or impact point locations are explained for final test execution. Section 4 summarizes the outcomes and results of final test cases. Finally, conclusions from the experiment are highlighted in Section 5.

\section{Safety index and experimental evaluation}

A summary of main existing safety indexes is outlined in this section. These are the formulations that are the most related ones to the herein proposed experiment procedure. The first four indexes belong to investigations for automotive fields, since those are based on a long experimental activity.

In the 60s Lissner introduced the Wayne State University Concussion Tolerance Curve (thereafter WSTC) [8] that represents the maximum acceptable acceleration versus time of impact. The failure criterion was the skull fracture and/or concussion in the head of cadavers that were tested against a flat surface. The resulting curve is based on three main parameters, such as acceleration, temporal pressure and time of impact in seconds. Later, Gurdjian and Patrick [9] improved the index, by using more experimental data. This is considered the foundation for most currently accepted head injury indexes.

The Gadd Severity Index [7] that was obtained from the WSTC is again an expression of the head acceleration response as function of the impact with pulse duration. In fact, $a$ is the average acceleration of the pulse of interest and $n$ is assumed in general as 2.5 [7,19], so that the index is expressed as:

$$
\operatorname{GSI}(a)=\int_{0}^{T} a^{n} d t .
$$

Values of GSI > 1.000 are considered to be dangerous for life (injury with un-survivable effect) [7]. 
The quantitative index most spread world-wide which takes into account the resultant of head translational acceleration, based on the same failure criteria as WSTC (skull fracture), is the HIC, the Head Injury Criterion [18] given in the form

$$
H I C\left(a_{h}\right)=\left[\frac{1}{t_{2}-t_{1}} \int_{t_{1}}^{t_{2}} a_{h} d t\right]^{2.5}\left(t_{2}-t_{1}\right)
$$

where $a_{h}$ (measured in g's-gravity acceleration ratio) is the resulting acceleration of the human head and $\left(t_{2}-t_{1}\right)$ (time measured in seconds) is selected so as to maximize HIC. Since $a_{h}$ is dimensionless, HIC is measured in seconds. HIC has been validated as a predictor for skull fracture and brain injury in a testing context by using blunt force trauma for an unconstrained head.

The HIC index is commonly used to quantify the head injury through reference values depending on the kind and size of humans in terms of male, female, child, etc. There are two different values of this criterion, namely $\mathrm{HIC}_{36}$ with $\left(t_{2}-t_{1}\right)=36$ ms and HIC ${ }_{15}$ with $\left(t_{2}-t_{1}\right)=15 \mathrm{~ms}$. According to Gao [20], among others, only $\mathrm{HIC}_{15}$ can be applicable to short impact times, since the injury probabilities are more restricted than for $\mathrm{HIC}_{36}$ [15].

The relation between HIC and commonly used risk injury evaluation, named as the Abbreviated Injury Scale (AIS) ${ }^{4}[15,20]$ is reported in Table 1 [21].

According to Haddadin [15], upper and lower limits for the injury risk are function of a certain probability of AIS $\geq 3$. Within this range $\mathrm{HIC}_{36}$ varies from 650 (that represent a $5 \%$ probability of serious injury) to 1000 (that implies a $20 \%$ probability of serious injury). Values below 650 represent very low injury risk according to the European New Car Assessment Programme (EuroNCAP) Protocol [22].

Since HIC does not consider the direction of impact and neglects angular accelerations, Newman [23] introduced an index named as Generalized Model for Brain Injury Threshold (GAMBIT), which involves both angular and linear accelerations $\left(\alpha_{\mathrm{res}}\right.$ and $\left.\mathrm{a}_{\mathrm{res}}\right)$ and is expressed as

$$
G(t)=\left[\left(\frac{a_{\text {res }}(t)}{250}\right)^{2}+\left(\frac{\alpha_{\text {res }}(t)}{25000}\right)^{2}\right]^{\frac{1}{2}}
$$

It applies for a $50 \%$ of probability of AIS $\geq 3$.

Within robot industry, approaches for safety factors can be found as follows.

Based on HIC, but considering a single rigid joint moving at uniform linear speed $v$ before the impact, the Safety Index for Robots (SIR) was established in $[10,12]$ as

$$
S I R=2\left(\frac{2}{\pi}\right)^{\frac{3}{2}}\left(\frac{K_{\text {cov }}}{M_{\text {oper }}}\right)^{\frac{3}{4}}\left(\frac{M_{T}}{M_{T}+M_{\text {oper }}}\right)^{\frac{7}{4}} v^{\frac{5}{2}}
$$

where $M_{T}$ is the total effective mass, as the sum of $M_{r}$ (moving mass of the robot) and $P$ (maximum payload); $M_{\text {oper }}$ is the weight of impacted operator and $K_{c o v}$ is stiffness of the contact surface. This criterion has been validated in applications with industrial robots, but not with service robots. One of the main limits of this index is that damping effects are not taken into account.

Based on the former index, a new analysis gave a new specific index to quantify the severity of head impact against robots. It is the New Index for Robots (NIR) [17]. The purposes of this index are to provide an easy calculation by using technical data that are provided in manufacturer catalogs and to understand easily the comparison with HIC. Its expression is given as

$$
N I R=2\left(\frac{2}{\pi}\right)^{\frac{3}{2}}\left(\frac{1}{9.81}\right)^{\frac{5}{2}}\left[\frac{K_{\text {cov }}}{M_{\text {oper }}}\right]^{\frac{3}{4}}\left[\frac{M_{T}}{M_{T}+M_{\text {oper }}}\right]^{\frac{7}{4}} v_{\max }^{\frac{5}{2}}
$$

with

$$
K_{\mathrm{cov}}=\left[\frac{E_{m} A_{m}}{L_{m}}\right]^{\frac{3}{4}}
$$

where $M_{T}$ again stands for the total effective mass; $v_{\max }$ is the maximum possible velocity of the robot considered; $K_{c o v}$ is stiffness of the contact surface; $E_{m}$ isYoung's modulus of the material of the robot arm; $A_{m}$ is the cross section and, $L_{m}$ is the arm length.

The advantage of NIR against HIC can be considered in its formulation that is based on parameters of robot design and not just on impact characteristics. The formulation of NIR is also more convenient than HIC formulation as per robot applications since it can be evaluated in a more fairly easy way since it uses robot characteristics that can be identified in a fairly easy procedure.

In order to compare NIR with HIC, $M_{\text {oper }}$ can be considered as $5 \mathrm{~kg}$ as for a head weight as an approximation of human anatomy mass. NIR is the index that is characterized experimentally in this paper. However, NIR can be used to estimate also other types of impacts, like robot against human arm or other parts of human body.

${ }^{4}$ AIS: The Abbreviated Injury Scale differentiates observed levels of injury into seven categories from none to fatal effects. It was developed by the Association for the Advancement of Automotive Medicine. 
Table 1

HIC vs AIS according to Prasad/Mertz curves [20].

\begin{tabular}{lll}
\hline & AIS range & Injury state \\
\hline HIC & $=0$ & No injury \\
HIC & $\geq 2$ & Skull fracture \\
HIC & $\geq 4$ & Brain injury \\
HIC & $=6$ & Unsurvivable \\
\hline
\end{tabular}

\section{Experimental layout}

The proposed experiment approach tries to validate NIR index by acquiring experimental data to confirm the soundness of its formulation by using a low-cost impact model. A set of impact tests that simulate the collision of a human head with the arm of an industrial robot was carried out at LARM in Cassino. Second goal of the experiment approach is to compare test outputs with results in applying HIC (Head Injury Criterion) by using measurements of the acceleration-time response of the head during tests.

A layout for collision tests has been setup by representing a general test situation as shown in the scheme of Fig. 1. This general layout takes into account all possible scenarios in industrial workplaces for the following cases: robots with several degrees of movement, represented by joints 1,2,3 and 4; different impact point locations on robot, such as P2, in the middle of link 2, PX at the end point of such link (and possibly the worst case) or H representing the payload hanging from the extreme of robot arm; different impact point locations on human (not only head can be affected, but also human arms can be injured). Even the position of both human and robot can be with dynamic aspects (robot or human in motion), so linear speed of robot or human must be added to the speed of robot arm at working conditions.

A general flow chart for this layout in Fig. 2 summarizes all parameters and surrounding restrictions that may have a relevant role in the collision between human and robot. It draws attention not only to parameters that are related to the robot motion specifications, but also to robot material specifications, characteristics of the head model, constraints of test bench and sensors for data collection. As shown in the flow chart boxes, different inputs for robot motion (speed, trajectory of movements, points of impact in robot arm, rated and maximum payload) allow identifying several possible configurations for an impact. A test configuration is conditioned by robot design parameters such as arm weight, stiffness and rigidity of joint or cover material and even characteristics of human head model like weight and clamping situation (the way in which the head is fixed to the test structure). In addition, damping can play an important role. Once a scenario has been defined and expected outcomes are defined, a setting up of sensors can be the next step in fixing sensors in proper locations.

\subsection{Experiment restrictions}

The robot for the experiment is a Scara Robot-Adept Cobra 600,4-axis robot that is used especially for packaging and material handling, with a fast and precise automation system (Fig. 3a), with a maximum payload of $5.5 \mathrm{~kg}$ and maximum radial reach of $600 \mathrm{~mm}$ (Fig. 3b). Technical specifications are provided in robot user's manual [24].

After analyzing the scenario and available means, limitations can be identified as:

- Even if maximum radial reach in the working area is $600 \mathrm{~mm}$ according to Fig. $3 \mathrm{~b}$, a limitation of the arm movement is due to robot size and the current position of the robot bedplate in the lab. This has forced to fix the position of the outer link (L2), so that the

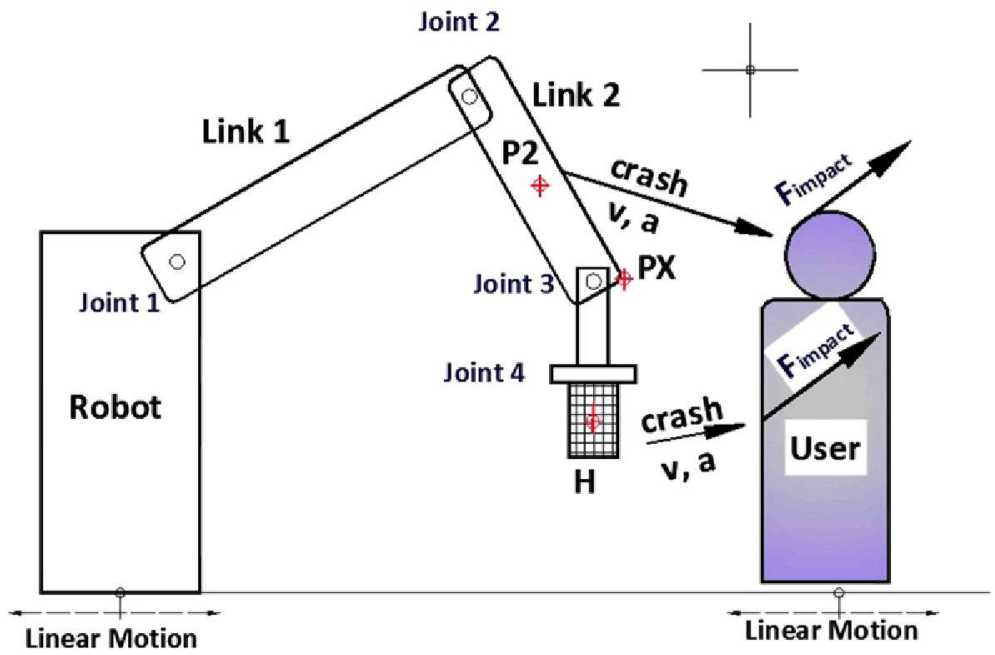

Fig. 1. A general layout simulating human-robot interaction with impact for lab tests. 


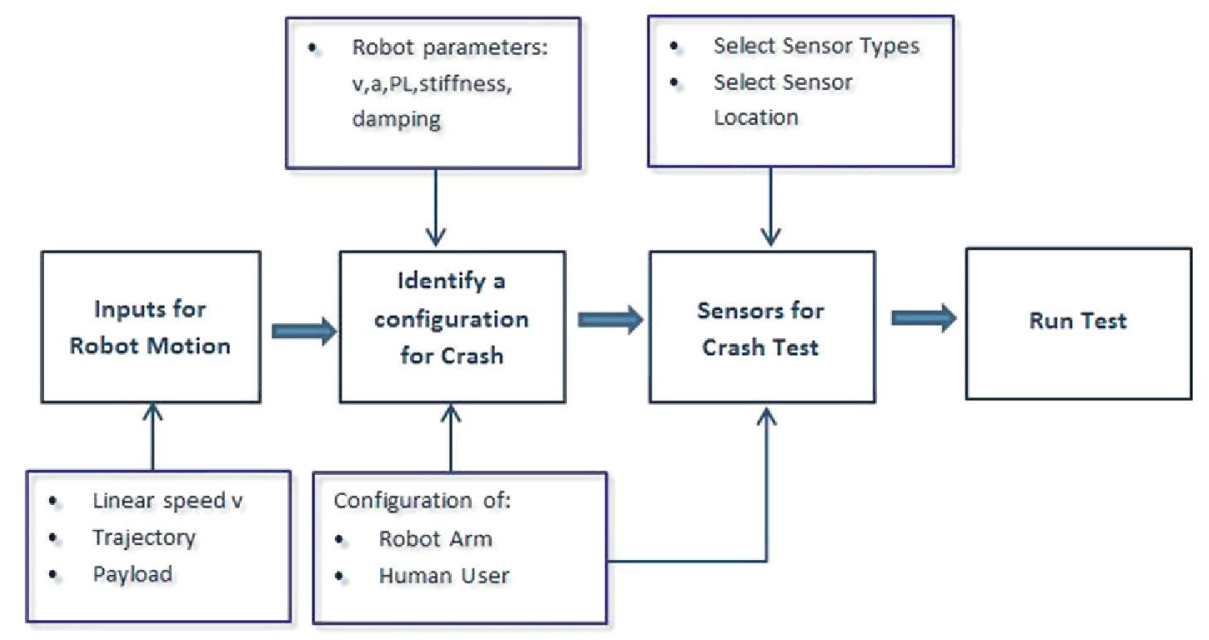

Fig. 2. A general flow chart for tests with experimental layout in Fig. 1.

inner link (L1) could not rotate, by affecting the final impact point for the crash. The maximum distance of $578 \mathrm{~mm}$ for displacement of P2 in the arm, as reported in Table 4 is due to robot size as indicated in the workspace in Fig. $3 \mathrm{~b}$ and to the available space for impact tests in the lab.

- The above considerations also contribute to consider the $v_{\max }$ as the maximum working speed for joint 1 so that according to robot specifications, $v_{\max }$ is $386^{\circ} / \mathrm{s}$.

- Arm is considered to be a rigid body system and damping effect is not considered.

\section{a}

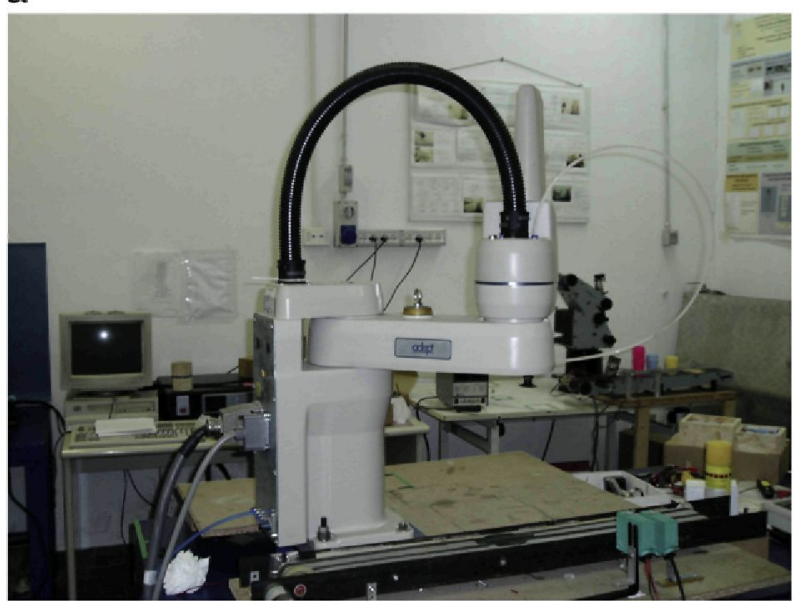

b

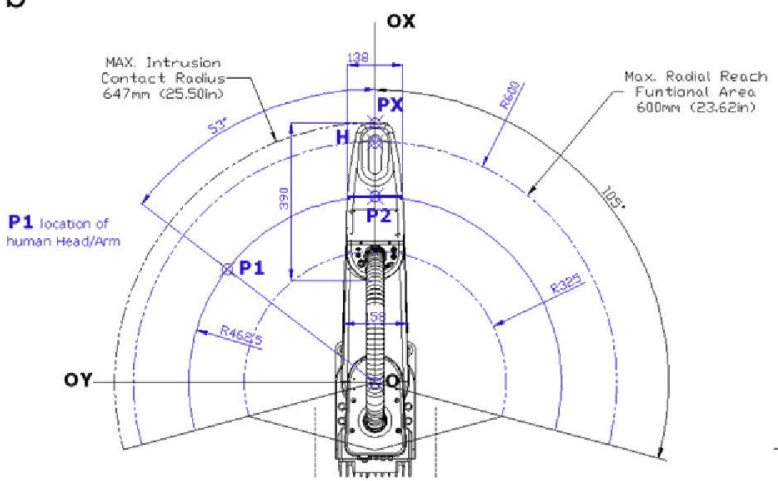

Fig. 3. a) The used robot Adept 1600 at LARM in Cassino; b) reach data of robot Adept 1600 . 
Because of these constrains and considering Eq. (2) for HIC safety index and Eq. (5) for NIR, six different scenarios have been setup as indicated in Table 2. The differences among tests conditions have been imposed in the positions of impact point on robot arm (linked directly to the length $\mathrm{L}_{\mathrm{m}}$ in NIR expression) and of the impact point on human model (head or arm).

It has been thought convenient to perform each test under five different speed conditions ( $10 \%, 20 \%, 30 \%, 40 \%$ and $50 \%$ of max working speed) in order to make a trend analysis. Each test has been repeated at least four times to guarantee statistical soundness of test results. Table 2 shows a summary of configurations for the six different test cases, where positions of impact points, both on robot and on human can be varied as indicated in Fig. 4a.

Among those points, impact point $\mathrm{H}$ has been neglected as due to space limitations in the laboratory area for the test and finally experiments have consisted of four scenarios with two possible impact locations P2 and PX as in Fig. 4b. Table 3 lists data for the locations of impact points according to robot coordinate system. The position of the impact point on robot (PX) represents the maximum radius of the available workspace, as shown in Fig. $4 \mathrm{a}$ and $\mathrm{b}$.

For the calculation of the total effective mass $\mathrm{M}_{\mathrm{T}}$, payload $\mathrm{P}$ has been neglected also because no extra weight was installed at the end-effector of the robot (point $\mathrm{H}$ in Fig. 4a).

Due to lack of information about the moving mass of robot (weight of inner and outer links) as from in the robot catalog, $\mathrm{M}_{\mathrm{r}}$ value has been estimated to be $20 \mathrm{~kg}\left(19.7365 \mathrm{~kg}\right.$ ) with a uniform ratio of mass/volume among the robot links. $\mathrm{A}_{\mathrm{m}}$ (averaged cross-section of robot) and $\mathrm{L}_{\mathrm{m}}$ (robot arm length) at impact point could be easily calculated as based on the experiment layout, but a lack of information has not permitted an estimation of Em (Young's modulus of the arm material). To overcome this problem, a value of $25,000 \mathrm{~N} / \mathrm{m}$ has been assumed for stiffness of contact surface $k_{\text {cov }}$ within final calculations of NIR, which was based on the estimation of HIC for assembly PUMA 560 robot in [20].

Although information on the robot arm characteristics was not completely known as from the available robot specification in [24], the formulation of NIR has permitted an interpretation of experimental tests with reasonable results, since the main focus is of the interaction between the robot end and human body parts.

\subsection{Software $\mathcal{E}$ hardware selected for testing}

Regarding software, LABVIEW 8.6 has been used for test measurement and control together with a data acquisition USB card of model NI 6009. Program file has been set up to acquire data every 15 ms according to [20] for HIC15 computation for short impact times.

A low-cost deformation sensor (Model ABA; $100 \mathrm{~kg}$-capacity; Trademark: TEMPO) has been used for impact force measurements (Fig. 5a). Two accelerometers, with 3-axis acceleration sensor boards MMA7260Q prototype PCB of SURE Electronics, (Fig. 5b), have been properly connected according to manufacturer guidelines and, after calibration they have been used for acceleration data acquisition. One was located at the back of the impact point in the human head model and the second one was placed at the back of the impact point in the robot arm, (Fig. 5c). In order to setup time at impact, a mechanical switch (Commutator PN4-K63 (15ª ; 125 / $250 \mathrm{~V})$ ) was also connected to the system.

A scheme of sensor connections both to the power supplies and the USB data acquisition card is described in Fig. 6.

Inputs of data acquisition card come from accelerometer no. 1 (fixed to head model), accelerometer no. 2 (fixed to back of robot arm), impact force sensor, and mechanical switch.

Regarding hardware, two different models have been used to simulate both head and human arm. The first one was a leather ball about $3 \mathrm{~kg}$ in weight filled with sand as shown in Fig. 7a. Ideal weight should have been $5 \mathrm{~kg}$ in order to get results comparable with those of HIC, but a restriction in the supporting structure avoided to increase weight more than $30.1 \mathrm{~kg}$. The second model for human arm was a plastic cylinder of $00.677 \mathrm{~kg}$ weight as shown in Fig. $7 \mathrm{~b}$.

The built model represents quite close the behavior of a human head under impact, because of its likeness in shape and weight to the head structure of the dummy family (a padded rigid aluminum shell) that is used generally in experimental testing for worldwide accepted $\mathrm{HIC}_{15}$ criterion. Neither of those models deform exactly as a human skull under loading.

Damping effect due to human head-neck link was also neglected in our experiments. But, this does not invalidate experiment outputs. Since according to existing literature [14] "kinematic behavior of the human head is independent from the neck stiffness for short time impacts $(t<10 \mathrm{~ms}$ )". A short impact time is exactly the type of impacts expected for the proposed experiments.

Because of above remarks, head model and its arrangement have been considered valid enough, although a recommendation for future experiments will require improvements of the model in terms of material, weight, stiffness and clamping conditions.

Table 2

Types and data for experimental tests.

\begin{tabular}{lll}
\hline TEST N & Position of impact point on robot $\left(\mathrm{L}_{\mathrm{m}}\right)$ & Position of impact point on human body \\
\hline 1 & $\mathrm{P} 2$ & Head \\
2 & $\mathrm{P} 2$ & Arm \\
3 & $\mathrm{H}$ & Head \\
4 & $\mathrm{H}$ & Arm \\
5 & $\mathrm{PX}$ & Head \\
6 & $\mathrm{PX}$ & Arm \\
\hline
\end{tabular}



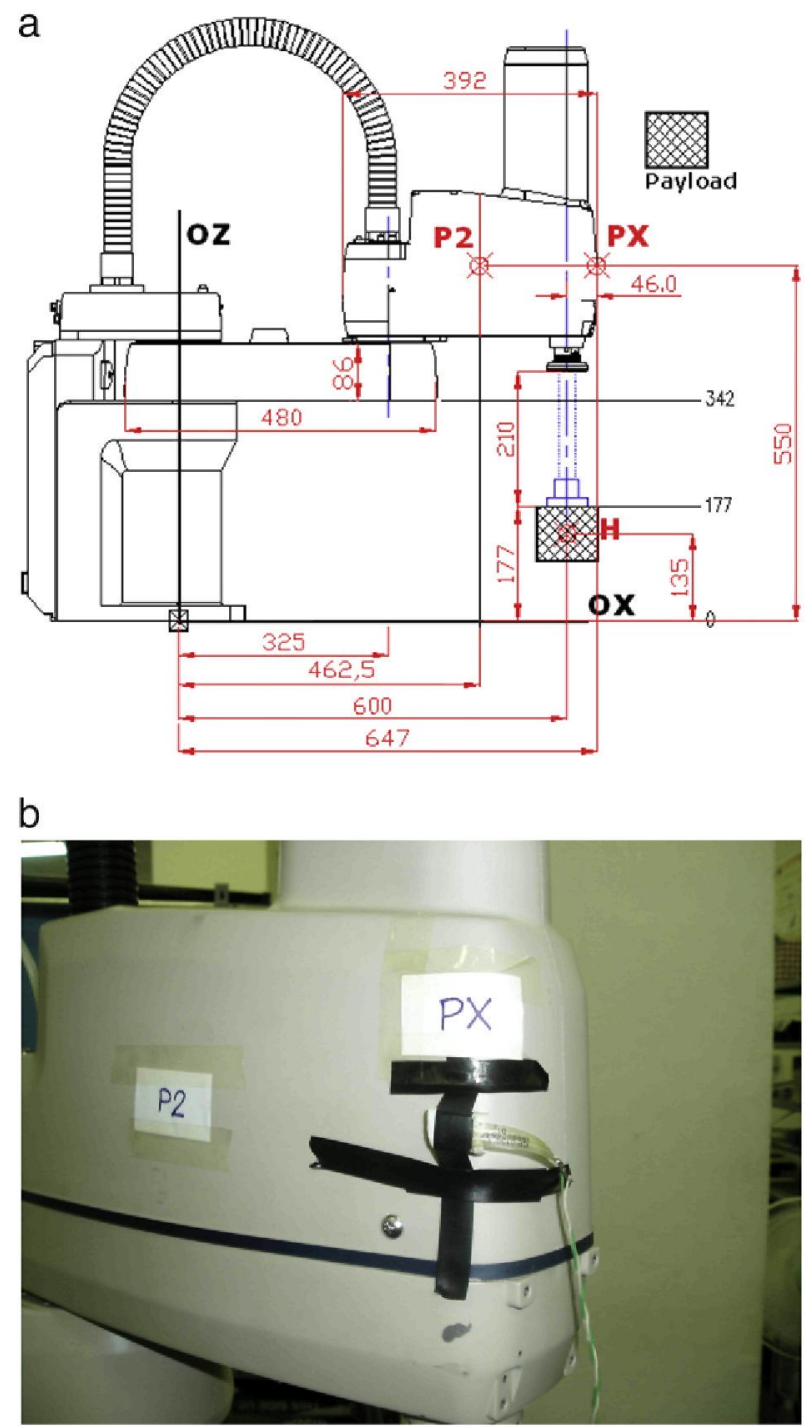

Fig. 4. Location of possible impact points on the robot: a) according robot reference system; b) on robot arm.

\section{Results of lab tests}

The test set-up in the lab in Cassino is shown in Fig. 8 for the case of the simulation experiment of head injury using an impact of robot arm against a sand-filled soccer ball.

Each one of the tests was repeated four times for statistics validation. Results are reported in Tables 4,5 and 7 referring to the tests Nos. 1, 5 and 6 in Table 2. The results of experiment No. 2 were finally not considered because problems of sliding and rebounding during the tests made the results not valid. The human arm model slipped along the robot arm during the crash and this avoided a proper data measure by the load sensor cell.

Before each test, position of the impact point on robot arm was adjusted to guarantee a proper record of the load sensor cell. This justifies that position of impact point P2 on robot shows limited variations in Test no. 1 (Table 4).

HIC has been calculated by taking account of the interval of time that maximizes the results of the integral in Eq. (2), instead of assuming a triangular wave response (pulse waveform) of the human head after impact by the robot, as indicated in [7].

\section{Table 3}

Locations of impact points with respects to coordinate system in Fig. 4a.

\begin{tabular}{llll}
\hline Impact point on robot & $X(\mathrm{~mm})$ & $\mathrm{Y}(\mathrm{mm})$ & $\mathrm{Z}(\mathrm{mm})$ \\
\hline P2 & 462.5 & 0 & 550 \\
PX & 647 & 0 & 550 \\
\hline
\end{tabular}




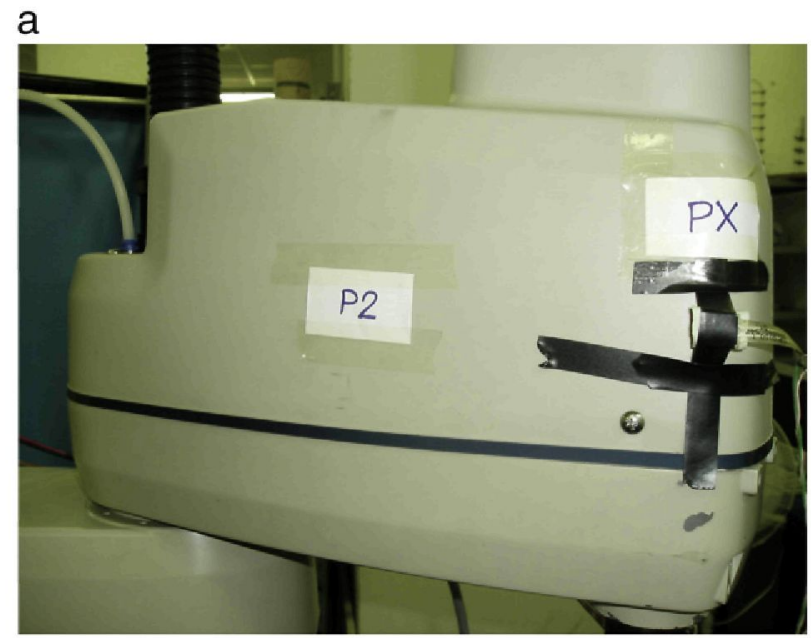

b

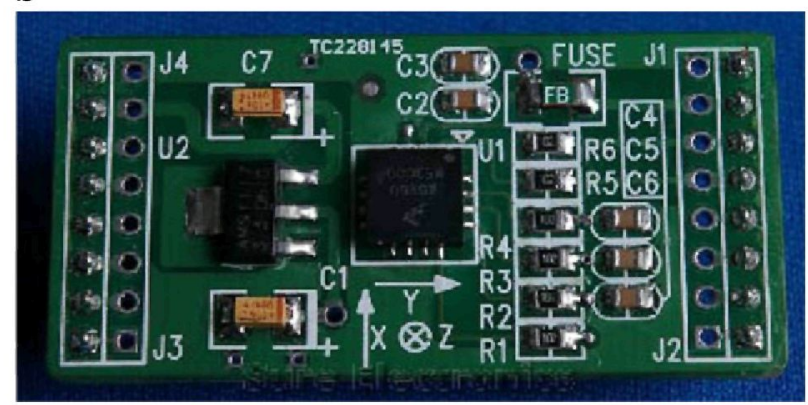

C

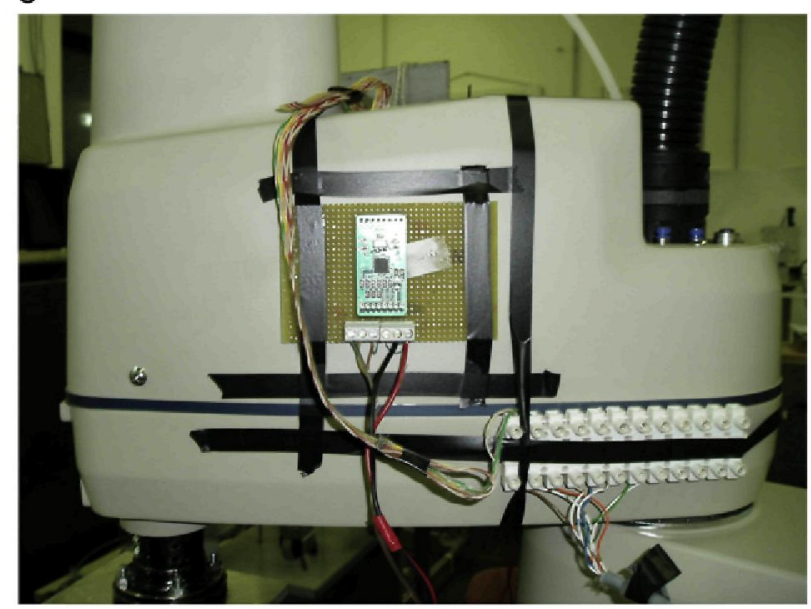

Fig. 5. Setup of sensors in experimental layout: a) load cell at PX impact point; b) 3-axis acceleration Sensor Board; c) accelerometer at the back of robot arm.

Focusing on the outputs of tests No. 1 and No. 5, where head model was used, results and plots confirm an expected evolution of both the HIC index and value of $\mathrm{a}_{\text {impact. }}$ as long as impact velocity increases. Even if the values of recorded acceleration 1 of test No. 1 as in Fig. 9, lead to an irregular plot of HIC as in Fig. 10, the average value of HIC for Test No. 1 gives an output as expected, namely the greater is the robot arm working speed, the greater is HIC, as reported in Fig. 11.

Similar comments can be worked out as regarding results of test No. 5 as shown in Fig. 12, although measurements of $a_{\text {impact }}$ show less dispersion than those from test No. 1.

If values of Average HIC are compared for Test No. 1 and Test No. 5, as in Table 6, there is a ratio of about 2.1 at any robot speed. This confirms the hypothesis that the position of impact point at the end of the robot cover (PX) is possibly the worst case in terms of human head safety risk. 


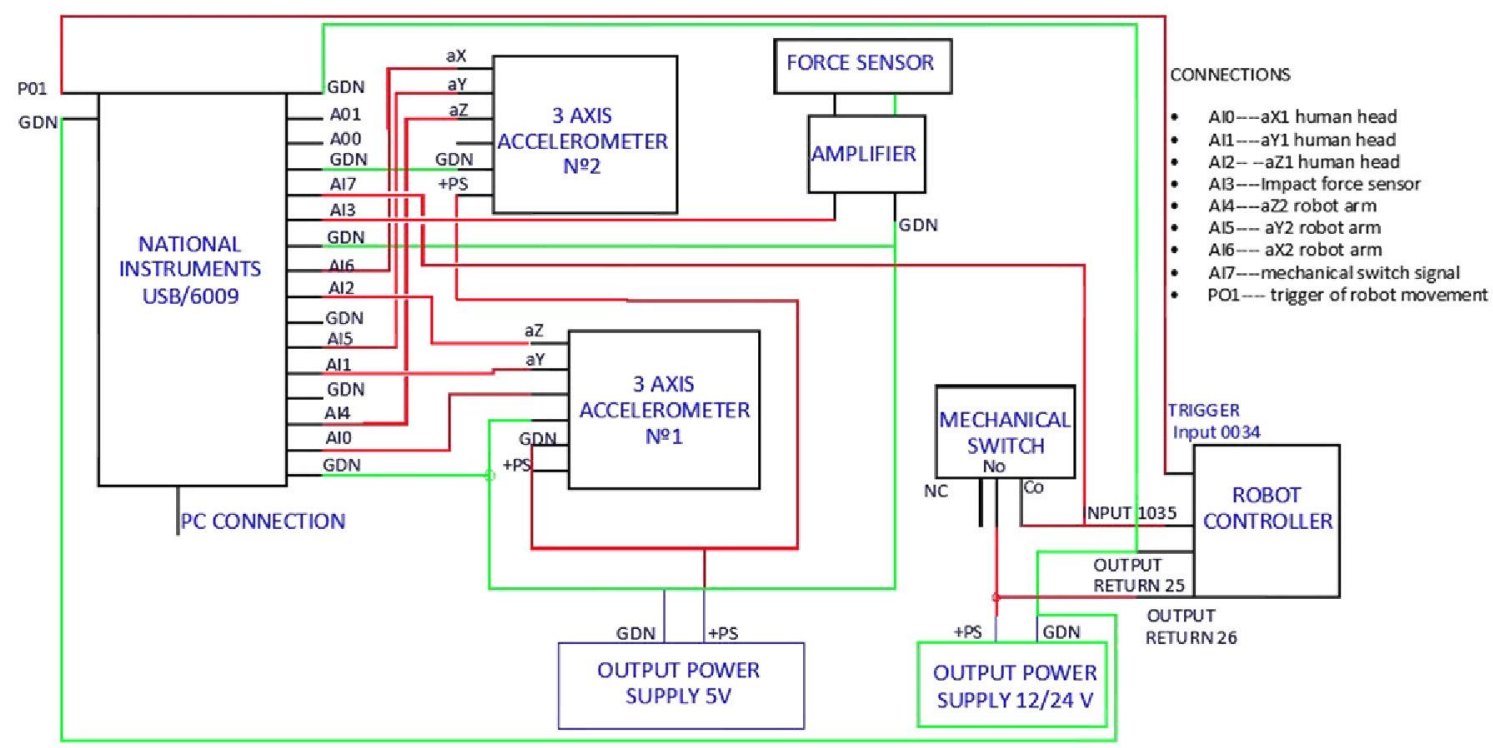

Fig. 6. A scheme of sensor connections in the experimental layout in Fig. 5.

When comparing values for new safety index NIR with values for Average HIC as in Table 6, there is a ratio of differences that is almost constant as long as robot working speed increases. That ratio varies from 300 times to 700 times as depending on the test No. But focusing on the trend analysis of both NIR and the average value of HIC (Figs. 11 and 14), it can be asserted that the evolution of both indexes is the same as long as working speed increases. Comparing graphs of HIC in tests No. 1 and No. 5 (Figs. 10 and 13 ) with

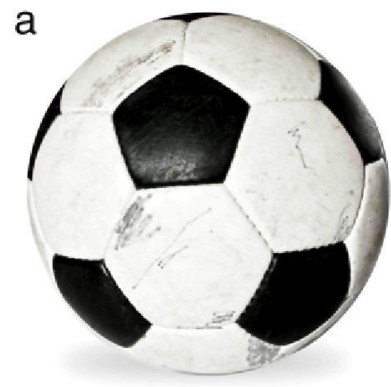

b

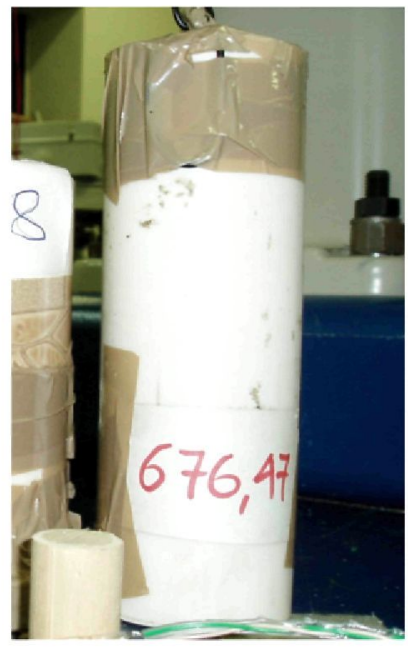

Fig. 7. Body models for testing: a) head model; b) arm model. 


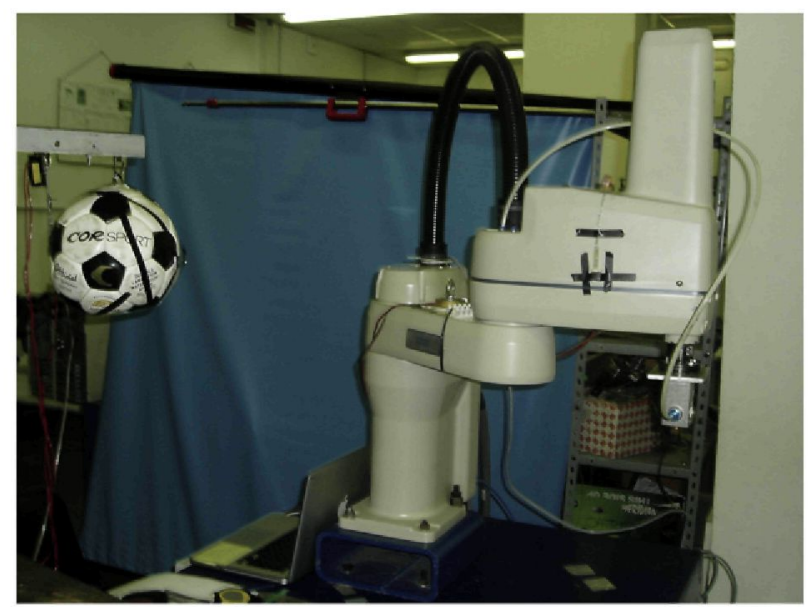

Fig. 8. A photo of the test-set in the laboratory in Cassino.

the experimental graphs (robot velocity in $\mathrm{m} / \mathrm{s}$ versus HIC) of impact test performed with LWRIII and KUKA KR3-SI in already mentioned experiments in $[3,16]$ it shows the same behavior at increasing impact velocity, even if values do not exactly match. This gives NIR a validated characterization as a suitable criterion.

The reasons behind aforementioned difference of magnitude could be directly linked to the facts that can be explained as follows. The value of $20 \mathrm{~kg}$ for the moving mass of the robot could have been overestimated, since taking into account testing at lab performed by Bicchi et al. [11] or, experimental setup performed by Haddadin [4,15], an arm lightweight of $1.5 \mathrm{~kg}-2 \mathrm{~kg}$ or the use of an $1 \mathrm{~kg}$ aluminum impactor could be a better approach. This is aligned directly with the proposal of different researchers that point at the reduction of both the inertia and weight of the robot manipulator as one of the solutions for decreasing the severity of impacts [25].

On the other hand, according to [3], HIC was evaluated for several robot masses up to $500 \mathrm{~kg}$ and experiments demonstrated that HIC saturates for increasing robot mass at each impact velocity. So, at some point increasing robot mass does not result in higher HIC anymore, although it has an evident influence to be considered in future experiments.

Rigidity and weight of the head model also have conditioned results. In the testing, a value of $30.1 \mathrm{~kg}$ has been used, but ideal weight for $\mathrm{M}_{\text {oper }}$ should be $5 \mathrm{~kg}$ in order to have results comparable with literature data for HIC. The way of clamping the head model may (head-neck link) affect rigidity. According to Haddadin in [15], experimental results of robot-dummy crash run under the same range of robot velocities gave HIC values from nearby $0(0.2 \mathrm{~m} / \mathrm{s})$ to almost $25(2 \mathrm{~m} / \mathrm{s})$. Thus, it seems that this constrain effect has an evident influence. But secondly, in the same reference [15], two types of head loadings are classified, namely direct interaction (when something collides directly to the head) and indirect interaction (when the load is transmitted through the head-neck link as due to sudden changes in torso motion). It is also pointed out that the potential danger is much higher in cases of direct interaction and indeed most of the criteria and indexes (as WSTC. HIC, AIS) are available as referring to direct interaction.

Table 4

Data and results for Test No. 1, where $\mathrm{a}_{\text {impact }}$ is measured at impact time (NR is for Not Recorded Value).

\begin{tabular}{|c|c|c|c|c|c|c|}
\hline Execution & $\mathrm{V}_{\mathrm{H}}[\mathrm{m} / \mathrm{s}]$ & P2 location (m) & $a_{\text {impact }}[\mathrm{g}]$ & $F_{\text {impact }}[N]$ & $\mathrm{HIC}$ & NIR \\
\hline \multirow[t]{5}{*}{1} & $0.3894(10 \% \max )$ & \multirow[t]{5}{*}{0.578} & NR & NR & NR & 0.2171 \\
\hline & $0.7788(20 \% \mathrm{Vmax})$ & & 0.3275 & 2.0317 & 0.001715 & 1.2282 \\
\hline & $1.1682(30 \% \operatorname{Vmax})$ & & 1.0040 & 7.6585 & 0.017521 & 3.3845 \\
\hline & $1.5575(40 \% \mathrm{Vmax})$ & & 0.3958 & NR & 0.001871 & 6.9478 \\
\hline & $1.9469(50 \% \mathrm{Vmax})$ & & 0.7825 & 17.2529 & 0.012936 & 12.1373 \\
\hline \multirow[t]{5}{*}{2} & $0.3800(10 \% \operatorname{Vmax})$ & \multirow[t]{5}{*}{0.564} & 0.1728 & NR & 0.000292 & 0.2042 \\
\hline & $0.7599\left(20 \% \mathrm{~V}_{\max }\right)$ & & 0.1996 & NR & 0.001350 & 1.1552 \\
\hline & $1.1399(30 \%$ Vmax $)$ & & 0.2447 & NR & 0.000250 & 3.1833 \\
\hline & $1.5198\left(40 \% V_{\max }\right)$ & & 0.7519 & 15.5634 & 0.004947 & 6.5347 \\
\hline & $1.8998\left(50 \% V_{\max }\right)$ & & 0.5700 & 2.2719 & 0.003493 & $\mathbf{1 1 . 4 1 5 6}$ \\
\hline \multirow[t]{5}{*}{3} & $0.3806(10 \% \max )$ & \multirow[t]{5}{*}{0.565} & 0.1114 & 9.6877 & 0.000216 & 0.2051 \\
\hline & $0.7613(20 \% \max )$ & & 0.3343 & 1.7901 & 0.001590 & 1.1603 \\
\hline & $1.1419(30 \% \mathrm{Vmax})$ & & 0.4927 & 5.0563 & 0.004183 & 3.1974 \\
\hline & $1.5225(40 \% \max )$ & & 0.9066 & 3.2203 & 0.009660 & 6.5637 \\
\hline & $1.9031(50 \% \mathrm{Vmax})$ & & 0.7806 & 4.0335 & 0.008195 & 11.4663 \\
\hline \multirow[t]{5}{*}{4} & $0.3806(10 \% \mathrm{Vmax})$ & \multirow[t]{5}{*}{0.565} & 0.2414 & NR & 0.000550 & 0.2051 \\
\hline & $0.7613(20 \% \mathrm{Vmax})$ & & 0.4402 & 1.3033 & 0.002672 & 1.1603 \\
\hline & $1.1419(30 \% \mathrm{Vmax})$ & & 0.7961 & 3.8028 & 0.006685 & 3.1974 \\
\hline & $1.5225(40 \% \max )$ & & 0.9170 & 10.0783 & 0.018484 & 6.5637 \\
\hline & $1.9031(50 \% \max )$ & & 1.1017 & 3.2203 & 0.033267 & 11.4663 \\
\hline
\end{tabular}


Table 5

Data and results for Test No. 5, where a impact is measured at impact time (NR is for Not Recorded Value).

\begin{tabular}{|c|c|c|c|c|c|c|}
\hline Execution & $\mathrm{V}_{\mathrm{H}}[\mathrm{m} / \mathrm{s}]$ & PX location (m) & $a_{\text {impact }}[\mathrm{g}]$ & $F_{\text {impact }}[\mathrm{N}]$ & $\mathrm{HIC}$ & NIR \\
\hline \multirow[t]{5}{*}{1} & $0.3725(10 \% \mathrm{Vmax})$ & \multirow[t]{5}{*}{0.553} & 0.3234 & 1.6689 & 0.000624 & 0.1944 \\
\hline & $0.7451(20 \% \mathrm{Vmax})$ & & 0.7164 & 10.1752 & 0.004579 & 1.0997 \\
\hline & $1.1176(30 \% \mathrm{Vmax})$ & & 1.0323 & 22.948000 & 0.010116 & 3.0304 \\
\hline & $1.4902(40 \% \mathrm{Vmax})$ & & 1.0728 & 22.1718 & 0.023299 & 6.2207 \\
\hline & $1.8627(50 \% \mathrm{Vmax})$ & & 1.5225 & 21.5066 & 0.059212 & 10.8671 \\
\hline \multirow[t]{5}{*}{2} & $0.3725(10 \% \mathrm{Vmax})$ & \multirow[t]{5}{*}{0.553} & 0.3361 & 10.7498 & 0.000628 & 0.1944 \\
\hline & $0.7451(20 \% \mathrm{~V} \max )$ & & 0.7200 & 17.5981 & 0.003076 & 1.0997 \\
\hline & $1.1176(30 \% \mathrm{Vmax})$ & & 0.9965 & 22.2963 & 0.005746 & 3.0304 \\
\hline & $1.4902(40 \% \mathrm{Vmax})$ & & 1.2478 & 24.3928 & 0.012586 & 6.2207 \\
\hline & $1.8627(50 \% \mathrm{~V} \max )$ & & 1.2306 & 22.2136 & 0.019163 & 10.8671 \\
\hline \multirow[t]{5}{*}{3} & $0.3725(10 \% \mathrm{~V} \max )$ & \multirow[t]{5}{*}{0.553} & 0.3313 & 4.9439 & 0.000605 & 0.1944 \\
\hline & $0.7451(20 \% \mathrm{~V} \max )$ & & 0.7132 & 17.8014 & 0.003666 & 1.0997 \\
\hline & $1.1176(30 \% \mathrm{~V} \max )$ & & 1.0624 & 22.7284 & 0.013956 & 3.0304 \\
\hline & $1.4902(40 \% \mathrm{~V} \max )$ & & 0.8980 & 20.7186 & 0.015322 & 6.2207 \\
\hline & $1.8627(50 \% \mathrm{Vmax})$ & & 1.4362 & 23.6556 & 0.021175 & 10.8671 \\
\hline \multirow[t]{5}{*}{4} & $0.3725(10 \% \mathrm{Vmax})$ & \multirow[t]{5}{*}{0.553} & 0.4609 & $\mathrm{NR}^{*}$ & 0.001694 & 0.1944 \\
\hline & $0.7451(20 \% \mathrm{Vmax})$ & & 0.7063 & 6.5964 & 0.004482 & 1.0997 \\
\hline & $1.1176(30 \% \mathrm{Vmax})$ & & 0.8837 & 8.0745 & 0.013098 & 3.0304 \\
\hline & $1.4902(40 \% \mathrm{Vmax})$ & & 0.6920 & 16.3923 & 0.003311 & 6.2207 \\
\hline & $1.8627(50 \% \mathrm{Vmax})$ & & 1.6059 & 22.5368 & 0.025685 & 10.8671 \\
\hline
\end{tabular}

* NR: Recorded.

As experiments described on this paper are focused on a comparison of NIR with these globally recognized indexes, that neglect the influence of neck-head link, the experiments' results can be considered valid.

Anyway, both issues (rigidity and weight) must be analyzed in future experiments.

Latest consideration regarding the results of the NIR/HIC ratio can refer to the value $25 \mathrm{kN} / \mathrm{m}$ of contact surface stiffness $\mathrm{K}_{\text {cov }}$ for robot cover considered in NIR calculation. In Bicchi experiment [11], a cover stiffness of even $5 \mathrm{kN} / \mathrm{m}$ for a soft rubber cover could be an acceptable value. Again, either a value of head and robot combined stiffness $K_{\text {cov }}$ or $E_{m}$ need a better estimation in future experiments.

A summary of results for Test No. 6 is reported in Table 7 and Figs. 15 to 17.

Outputs of HIC as in Fig. 14 and recorded values of $a_{\text {impact }}$ show lower repeatability for the different executions than those from tests No. 1 and No. 5, where head model was used.

Executions 3 and 4 in Table 7 seem to have more realistic results since value of acceleration increases as long as impact speed increases, although $\mathrm{a}_{\text {impact }}$ at highest working speed shows a deviation from its trend as shown in Fig. 16. This affects also the value of average HIC and its plot as indicated in Fig. 17. A possible explanation of this issue is inherently related to the used model. During the experiments the arm model showed a different behavior in terms of clamping and damping than the head model. It was more unstable and there was a continuous need to relocate the model after the impacts due to the bounce effect.

Test outputs also confirm the expected problem in capturing impact force at load sensor with the arm model, with a larger number of Not Recorded (NR) measures.

When comparing values of new safety index NIR with values of Average HIC as in Table 8, the difference decreases to 80 times. But if value for $50 \% v_{\max }$ is underestimated, the ratio decreases to 13 times.

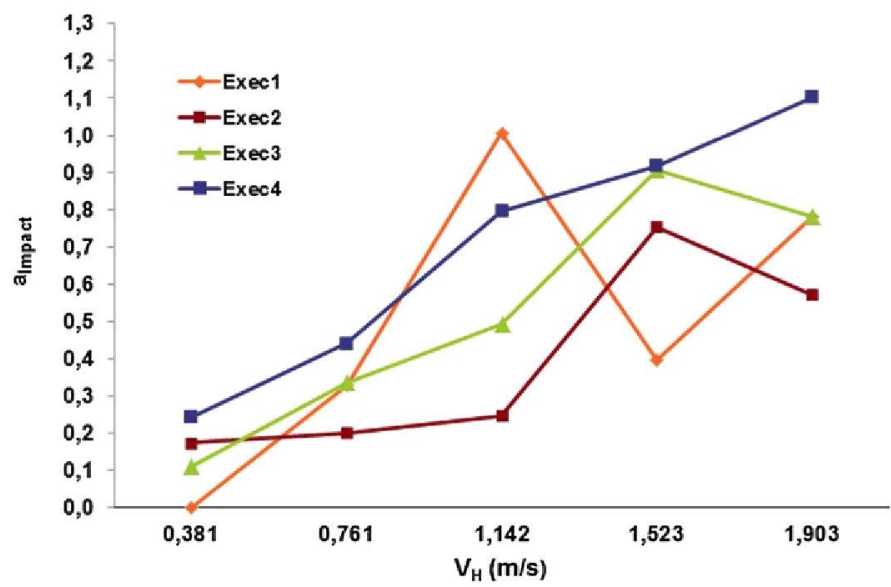

Fig. 9. Experimental head acceleration measures ( $\mathrm{a}_{\text {impact }}$ ) for Test No. 1 as function of the execution repetition, Table 4. 


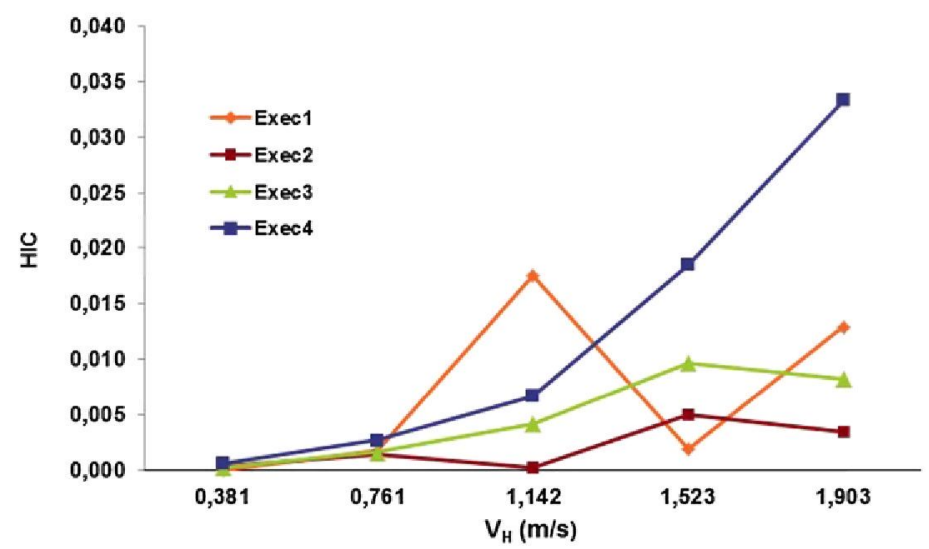

Fig. 10. Values of HIC for Test No. 1 as function of the execution repetition, Table 4.

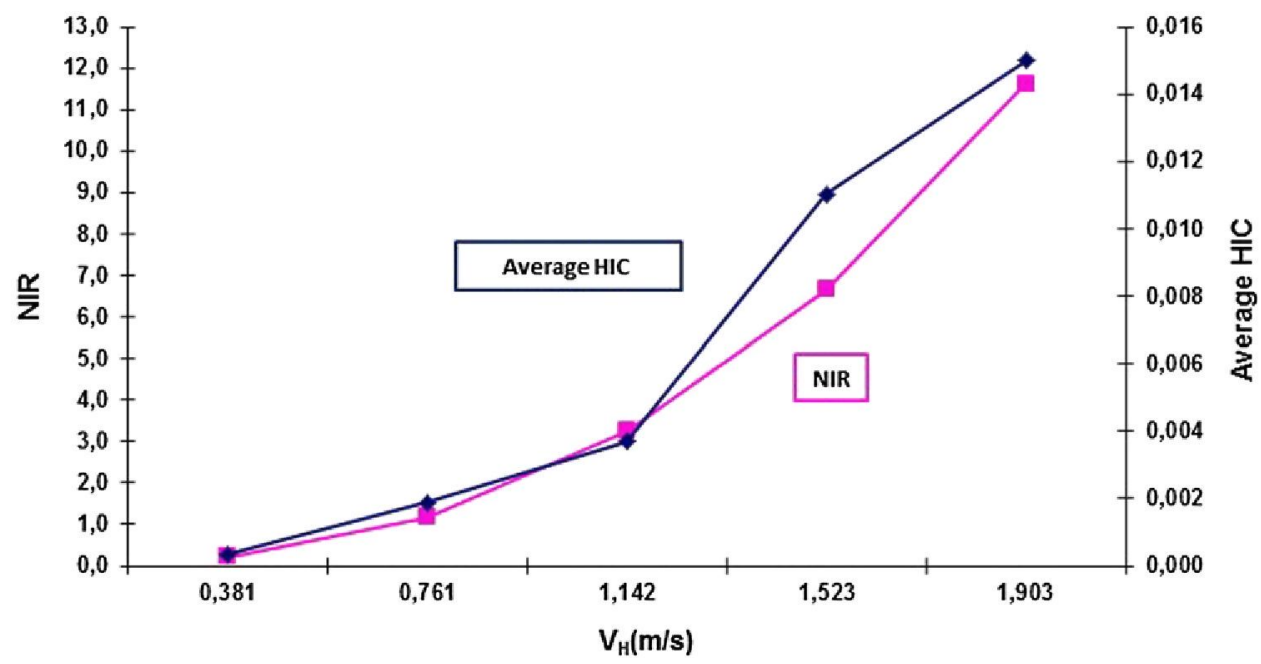

Fig. 11. Comparative analysis of NIR and Average HIC for Test No. 1 as function of impact speed.

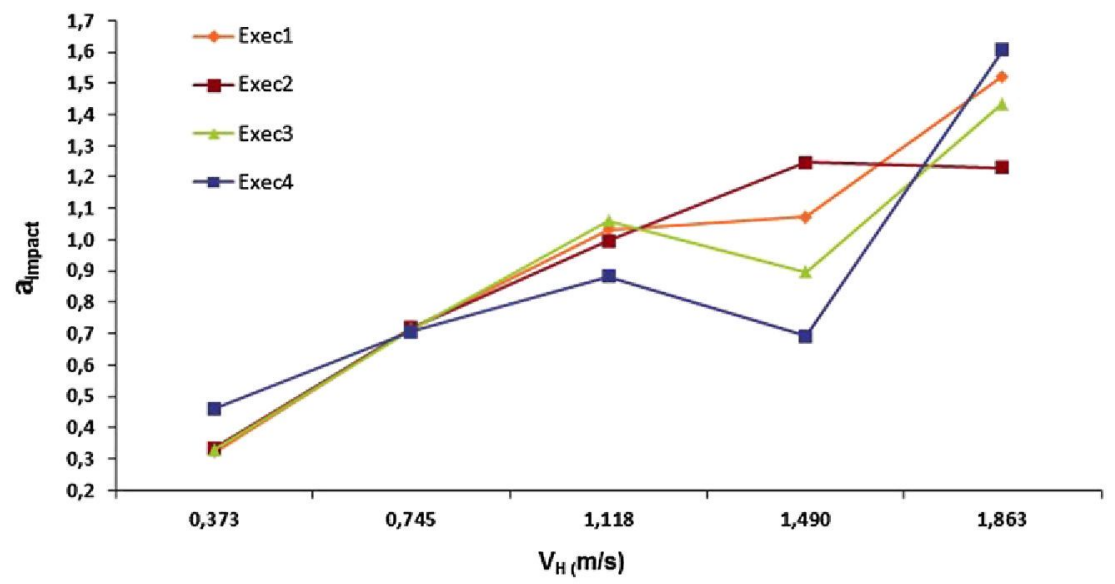

Fig. 12. Experimental head acceleration measures ( $\mathrm{a}_{\text {impact }}$ ) in Test No. 5 as function of the execution repetition, Table 5. 
Comparison of experimental results for HIC in tests with head model.

\begin{tabular}{|c|c|c|c|c|c|c|}
\hline \multirow[b]{2}{*}{$V_{H}[\mathrm{~m} / \mathrm{s}]$} & \multicolumn{3}{|l|}{ TEST No. 1} & \multicolumn{3}{|l|}{ TEST No. 5} \\
\hline & Average HIC & NIR & NIR/average HIC & Average HIC & NIR & NIR/average HIC \\
\hline $10 \% \max$ & 0.00035 & 0.2079 & 589.5 & 0.00089 & 0.194 & 218.9 \\
\hline $20 \% \max$ & 0.00187 & 1.1760 & 628.7 & 0.00395 & 1.100 & 278.4 \\
\hline $30 \% \max$ & 0.00371 & 3.2407 & 874.4 & 0.01073 & 3.030 & 282.4 \\
\hline $40 \% \max$ & 0.01103 & 6.6525 & 603.1 & 0.01363 & 6.221 & 456.4 \\
\hline $50 \% \max$ & 0.01499 & 11.6214 & 775.5 & 0.03131 & 10.867 & 347.1 \\
\hline
\end{tabular}

Although limited in number and with some differences, the performed tests have been considered good enough for indicating main behavior of the experiments with a physical interpretation of the results as a suitable characterization of the proposed safety index.

\section{Conclusions}

In this paper an experimental procedure has been proposed to evaluate safe issues for human-robot interaction with impacts by using a fairly simple lab layout with low-cost models and equipment. An experimental procedure has been outlined for the new index

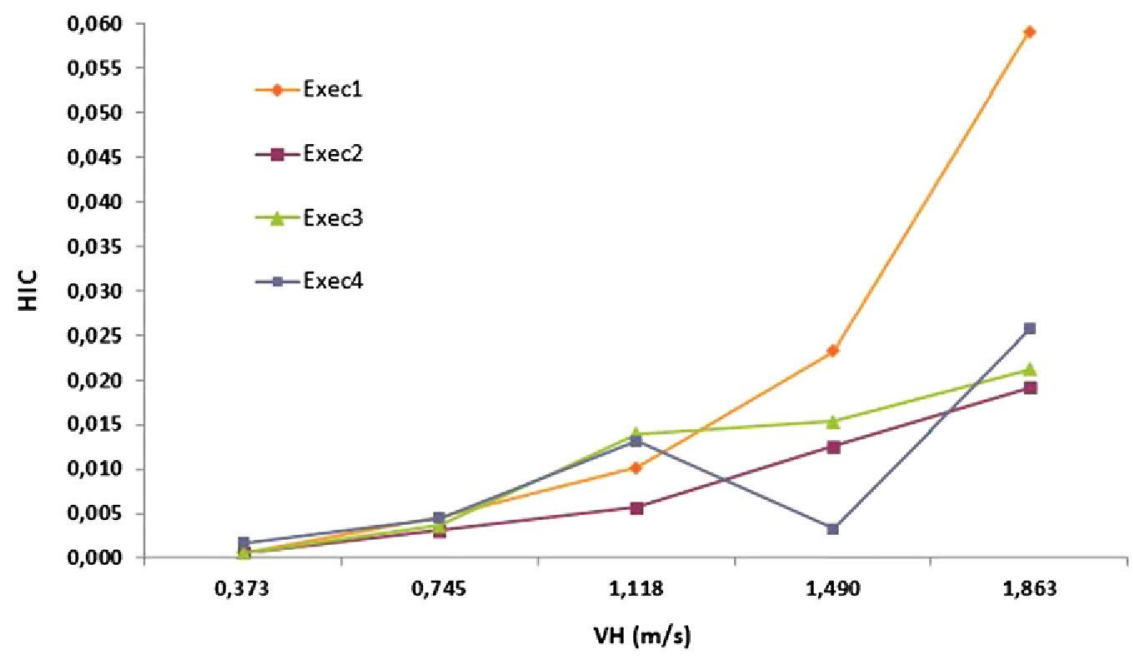

Fig. 13. Values of HIC for Test No. 5 as function of execution repetitions, Table 5.

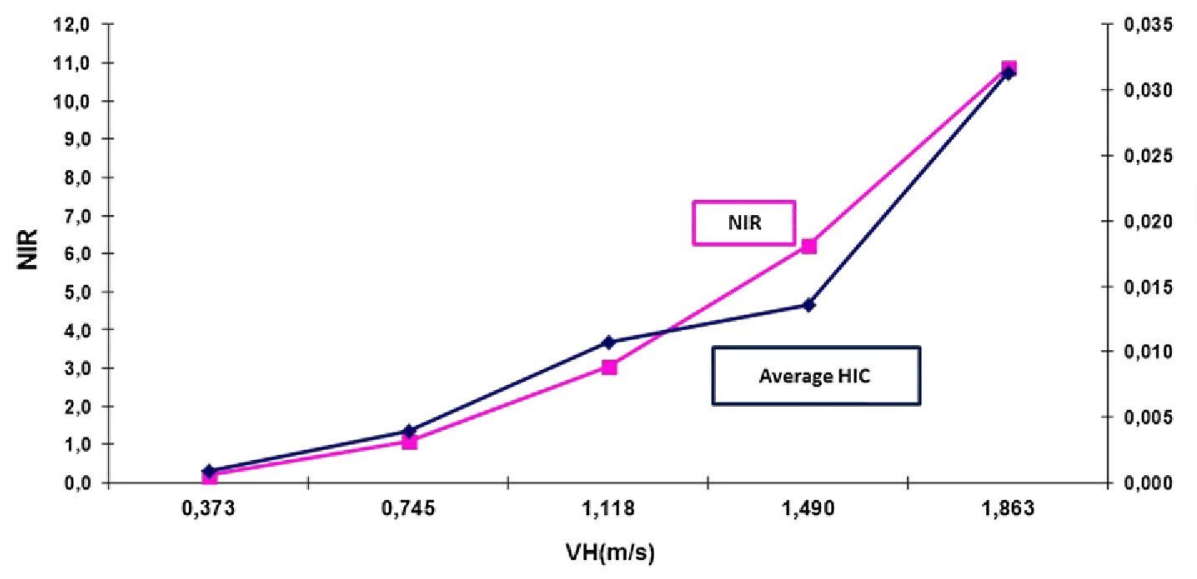

Fig. 14. Comparative analysis of NIR and Average HIC for Test No. 5 as function of impact speed. 
Table 7

Data and results for Test No. 6, where a impact is measured at impact time (NR is for Not Recorded Value).

\begin{tabular}{|c|c|c|c|c|c|c|}
\hline Execution & $\mathrm{V}_{\mathrm{H}}[\mathrm{m} / \mathrm{s}]$ & PX location (m) & $a_{\text {impact }}[\mathrm{g}]$ & $F_{\text {impact }}[N]$ & $\mathrm{HIC}$ & NIR \\
\hline \multirow[t]{5}{*}{1} & 0.3793 (10\%Vmax) & \multirow[t]{5}{*}{0.563} & 0.8633 & $\mathrm{NR}^{*}$ & 0.00451 & 0.2033 \\
\hline & $0.7586(20 \% \operatorname{Vmax})$ & & 4.2774 & 11.0328 & 0.23304 & 1.1501 \\
\hline & $1.1378(30 \% \operatorname{Vmax})$ & & 1.1246 & $\mathrm{NR}$ & 0.01328 & 3.1692 \\
\hline & $1.5171(40 \% \operatorname{Vmax})$ & & 1.3248 & $\mathrm{NR}$ & 0.02059 & 6.5058 \\
\hline & 1.8964 (50\%Vmax) & & 2.1176 & $\mathrm{NR}$ & 0.05285 & 11.3651 \\
\hline \multirow[t]{5}{*}{2} & $0.3793(10 \% \operatorname{Vmax})$ & \multirow{5}{*}{0.563} & 0.4649 & $\mathrm{NR}$ & 0.00178 & 0.2033 \\
\hline & $0.7586(20 \% \mathrm{Vmax})$ & & 0.5554 & $\mathrm{NR}$ & 0.02374 & 1.1501 \\
\hline & $1.1378(30 \% \operatorname{Vmax})$ & & 1.3798 & $\mathrm{NR}$ & 0.02125 & 3.1692 \\
\hline & $1.5171(40 \% \mathrm{~V} \max )$ & & 1.5186 & 21.0733 & 0.03211 & 6.5058 \\
\hline & $1.8964(50 \% \mathrm{Vmax})$ & & 1.3140 & $\mathrm{NR}$ & 0.02360 & 11.3651 \\
\hline \multirow[t]{5}{*}{3} & $0.3793(10 \% \mathrm{Vmax})$ & \multirow[t]{5}{*}{0.563} & 0.3766 & $\mathrm{NR}$ & 0.00108 & 0.2033 \\
\hline & $0.7586(20 \% \max )$ & & 1.4133 & 1.3033 & 0.01382 & 1.1501 \\
\hline & $1.1378(30 \% \operatorname{Vmax})$ & & 6.4414 & 19.4027 & 0.60067 & 3.1692 \\
\hline & $1.5171(40 \% \operatorname{Vmax})$ & & 6.8759 & 15.5868 & 0.68164 & 6.5058 \\
\hline & $1.8964(50 \% \mathrm{Vmax})$ & & 1.3635 & $\mathrm{NR}$ & 0.03750 & 11.3651 \\
\hline \multirow[t]{5}{*}{4} & $0.3793(10 \% \operatorname{Vmax})$ & \multirow[t]{5}{*}{0.563} & 0.2865 & $\mathrm{NR}$ & 0.00117 & 0.2033 \\
\hline & $0.7586(20 \% \operatorname{Vmax})$ & & 3.3568 & 21.6453 & 0.13369 & 1.1501 \\
\hline & $1.1378(30 \% \operatorname{Vmax})$ & & 5.8182 & 23.4871 & 0.45412 & 3.1692 \\
\hline & 1.5171 (40\%Vmax) & & 6.6236 & 22.1425 & 0.76540 & 6.5058 \\
\hline & $1.8964(50 \% \operatorname{Vmax})$ & & 1.8777 & NR & 0.05986 & 11.3651 \\
\hline
\end{tabular}

* NR: Recorded.

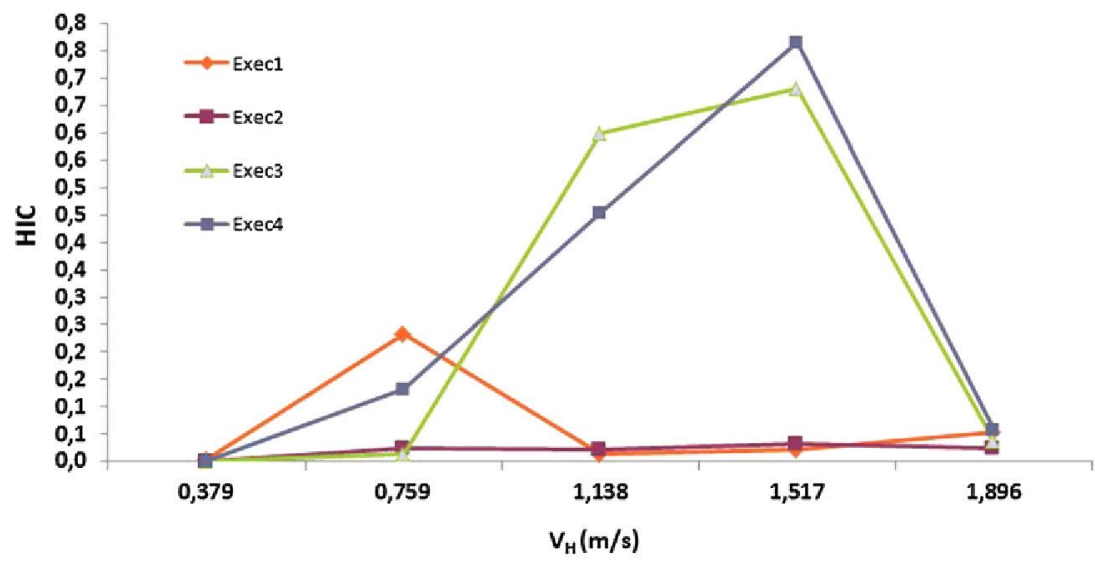

Fig. 15. Values of HIC for Test No. 6 as function of the execution repetition, Table 7.

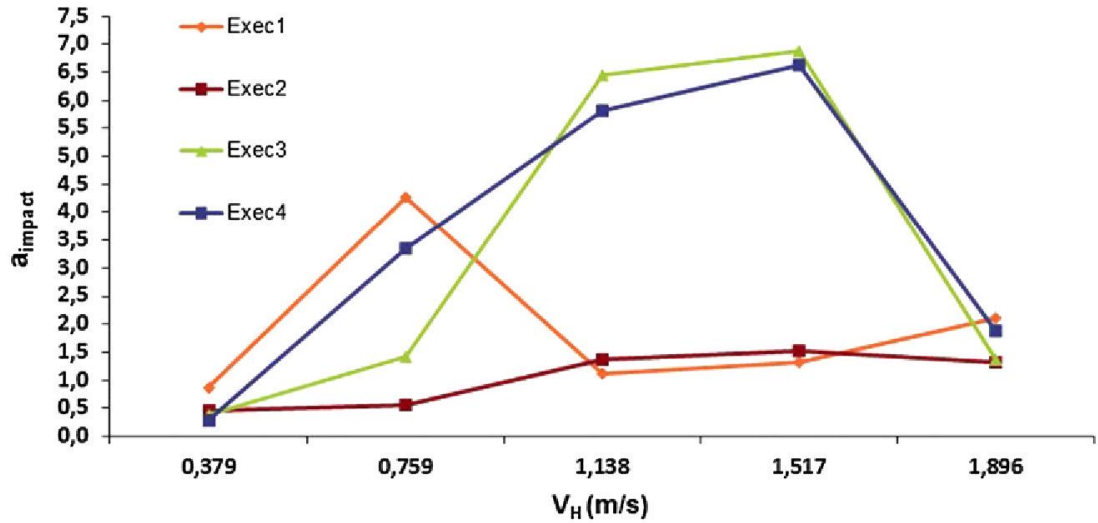

Fig. 16. Experimental head acceleration measures ( $\mathrm{a}_{\text {impact }}$ ) in Test No. 6 as function of the execution repetition, Table 7. 


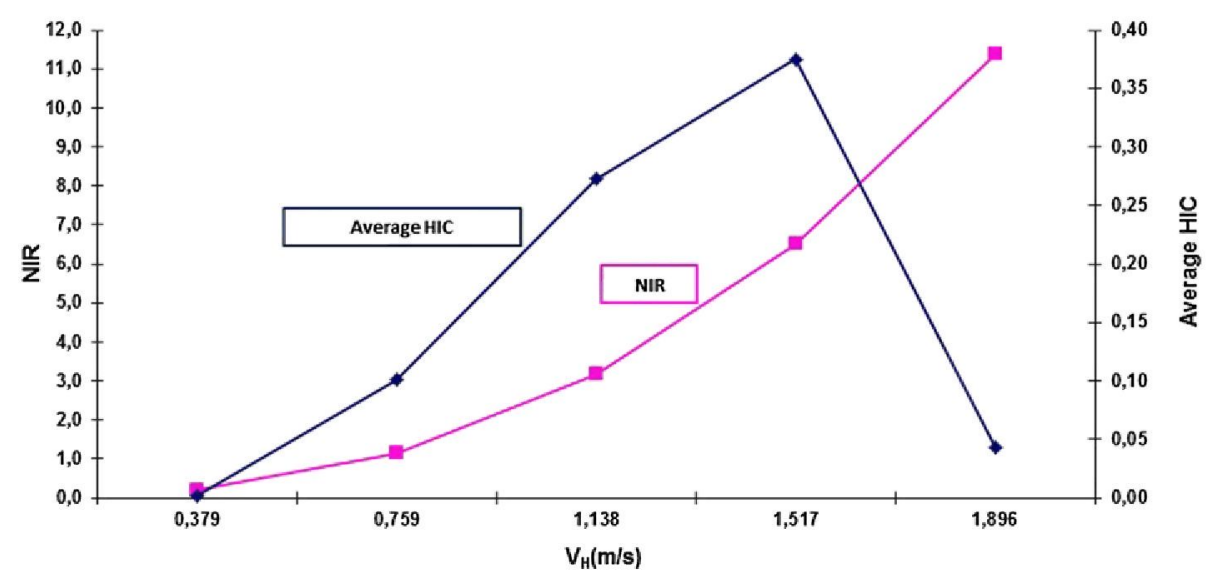

Fig. 17. Comparative analysis of NIR and Average HIC for Test No. 6 as function of impact speed.

Table 8

Comparison of results with experimental arm model for HIC index evaluation.

\begin{tabular}{llrr}
\hline & TEST No. 6 & & NIR \\
\cline { 2 - 4 } $\mathrm{V}_{\mathrm{H}}[\mathrm{m} / \mathrm{s}]$ & Average HIC & 0.2033 & NIR/average HIC \\
\hline $10 \% \max$ & 0.0021 & 1.1501 & 95.3 \\
$20 \% \max$ & 0.1011 & 3.1692 & 11.4 \\
$30 \% \max$ & 0.2723 & 6.5058 & 11.6 \\
$40 \% \max$ & 0.3749 & 11.3651 & 17.4 \\
$50 \% \max$ & 0.0435 & 261.6 \\
\hline
\end{tabular}

NIR by using data also from robot performance in user manuals. A validation campaign is reported with tests for robot impacts against models of human head and arm, whose results have been also used for HIC evaluation that has validated the soundness of the results.

Repeatability of test outputs proves the soundness of the proposed test procedure by pointing at the experimental techniques of reproducing the impact collision scenario as a proper way to evaluate safety indexes for robots, even when using a simple and lowcost model as the ones used in the reported experiments.

A comparison of computed values of HIC and NIR with the reported test results gives significance of NIR formulation as suitable for synthetic representation of a safety criterion both for analysis and design purposes.

The influence of main foreseen parameters and surrounding restrictions in the crash interaction, such as weight, stiffness and clamping of human models, type and arrangement of load sensor or the data acquisition time gap, is also highlighted and allow pointing out to improvement lines for future investigation. 